\title{
MicroRNA-26a acts as a tumor suppressor inhibiting gallbladder cancer cell proliferation by directly targeting HMGA2
}

\author{
HUADING ZHOU ${ }^{1}$, WEIJIE GUO ${ }^{2}$, YINGJUN ZHAO ${ }^{2}$, YIFEI WANG ${ }^{1}$, RUOPENG ZHA $^{2}$, JIE DING ${ }^{2}$,

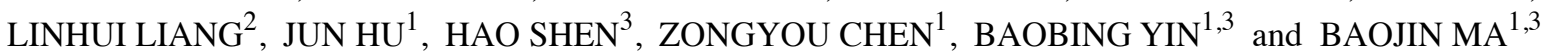 \\ ${ }^{1}$ Department of General Surgery, Huashan Hospital, Fudan University, Shanghai 200040; \\ ${ }^{2}$ State Key Laboratory of Oncogenes and Related Genes, Shanghai Cancer Institute, Shanghai Jiao Tong University, \\ School of Medicine, Shanghai 200032; ${ }^{3}$ Jing'an District Center Hospital of Shanghai Huashan Hospital, \\ Fudan University, Jing'an Branch, Shanghai 200040, P.R. China
}

Received January 16, 2014; Accepted March 13, 2014

DOI: $10.3892 / \mathrm{ijo} .2014 .2360$

\begin{abstract}
MicroRNAs (miRNAs) are a class of small, single-stranded, non-coding RNA molecules which can act as oncogenes or tumor suppressor genes in human cancer. However, the possible functions and mechanisms of miRNA action in gallbladder cancer (GBC) have not been elucidated. In the present study, it was found that miR-26a was often downregulated in GBC and the expression of miR-26a was associated with neoplasm histological grade. miR-26a significantly inhibited the proliferation of GBC cells based on the gain-of-function assays. Furthermore, we demonstrated that high mobility group AT-hook 2 (HMGA2) was a direct target of miR-26a. The results showed that HMGA2 mRNA levels and miR-26a levels were negatively correlated. In addition, we confirmed that reintroduction of HMGA2 antagonized the inhibition of miR-26a to GBC cell proliferation and all these effects were achieved through the cell cycle. Together, all these results suggest that miR-26a expression contributes to GBC proliferation by targeting HMGA2. miR-26a shows promise as a prognosis factor and therapeutic target of GBC patients.
\end{abstract}

\section{Introduction}

MicroRNAs (miRNAs) are a class of small, endogenous, noncoding RNAs which act as post-trancriptional regulators via binding to the $3^{\prime}$ untranslated regions (3'-UTRs) of the target gene mRNA, and have vital functions in many of physiological

Correspondence to: Dr Baojin Ma or Dr Baobing Yin, Department of General Surgery, Huashan Hospital, Fudan University, No. 12 Central Wulumuqi Road, Shanghai 200040, P.R. China

E-mail: macswq@126.com

E-mail: yinbaobing@126.com

Key words: miR-26a, tumor suppressor, gallbladder cancer, proliferation, HMGA2 and pathological processes, as well as in carcinogenesis (1-5). It has been reported that miRNAs can act as oncogene or tumor suppressor gene in various cancers, including lung, colorectal, and liver cancer $(6,7)$.

Gallbladder cancer (GBC) with poor prognosis is one of the most prevalent and aggressive malignant types of cancer in China. GBC is the tenth most common cancer in Shanghai and the average survival of patients is $\sim 9$ months (8). Many patients have no chance to accept radical operation when they are diagnosed. Despite its poor prognosis and high morbidity, the miRNA function in GBC remains largely unknown.

The miRNA microarray assays performed on 4 pairs of GBC and paracancerous tissues, revealed that miR-26a is significantly downregulated in GBC tissues. It has been reported that miR-26a was downregulated in pancreatic, nasopharyngeal, breast and lung cancer, and miR-26a can acts as tumor suppressor gene via directly targeting enhancer of Zeste homolog 2 (EZH2) (9-12). Also in hepatocellular carcinoma cells, miR-26a can inhibit cell proliferation by regulating cyclin D2, E2 and IL-6 $(13,14)$. It has been demonstrated that expression levels of miR-26a are lower in the tumors of renal cell carcinoma patients who developed tumor relapse, moreover, the lowest levels of miR-26a are observed in primary metastatic tumors (15). These findings suggested that miR-26a might play a vital role in GBC physiological and pathological processes.

In the present study, we demonstrated that miR-26a is significantly downregulated in GBC tissues compared with paracancerous tissues and miR-26a is closely correlated with the histologic grade of the neoplasm. Enforced, highly expressed miR-26a was able to inhibit GBC cell proliferation via cell cycle G1/S arrest. We verified that the high mobility group AT-hook 2 (HMGA2) was the direct and functional target of miR-26a.

\section{Materials and methods}

Clinical specimens. GBC and the matched paracancerous gallbladder tissues ( $2 \mathrm{~cm}$ from the tumor) were obtained from surgical specimen from Huashan Hospital (Fudan University, Shanghai, China). Diagnosis of each case was confirm by 
Table I. The primers used in the experiment.

\begin{tabular}{|c|c|c|}
\hline Gene & Use & $\left(5^{\prime}-3^{\prime}\right)$ \\
\hline Pri-miR-26a & PCR & $\begin{array}{l}\text { F: ACTAGTCAGAGCAAGACTCGGCAGGGTGTCTG } \\
\text { R: GTCGACCACCAGGCTTCCAATGGATCAGTGGTC }\end{array}$ \\
\hline HMGA2-ORF & PCR & $\begin{array}{l}\text { F: CCGGAATTCATGAGCGCACGCGGTGAGG } \\
\text { R: CGCGGATCCCTAGTCCTCTTCGGCAGACTCTTGT }\end{array}$ \\
\hline HMGA2-3'-UTR & PCR & $\begin{array}{l}\text { F: CCGCTCGAGGTGCCCTTTGTGTGTTCCAGGAGGA } \\
\text { R: ATGTACGCGGCCGCCAAGATGTGTGCAAGGGGTG }\end{array}$ \\
\hline HMGA2-3'-UTR-MUT1 & PCR & $\begin{array}{l}\text { F: TTTTCGTATAATCTTGTAGACACTTTGAACTTGATTTTTAACT } \\
\text { R: TTAGAAATAAAAAGTTAAAAATCAAGTTCAAAGTGTCTACAAG }\end{array}$ \\
\hline HMGA2-3'-UTR-MUT2 & PCR & $\begin{array}{l}\text { F: GCTTCTCTGCTAGATTTCTACATTATGAACTAAATTTTTTAACCA } \\
\text { R: GGAGCGACTTGGTTAAAAAATTTAGTTCATAATGTAGAAATCT }\end{array}$ \\
\hline HMGA2-3'-UTR-MUT3 & PCR & $\begin{array}{l}\text { F: AAATAAAAGCCAACCTTCAAAGAATGAACTAGCTTTGTAGGTG } \\
\text { R: CTTGTTGCATCTCACCTACAAAGCTAGTTCATTCTTTGAAGGT }\end{array}$ \\
\hline HMGA2 & Real-time PCR & $\begin{array}{l}\text { F: GAAGCAGCAGCAAGAACC } \\
\text { R: CCAGTGGCTTCTGCTTTC }\end{array}$ \\
\hline GAPDH & Real-time PCR & $\begin{array}{l}\text { F: TCTGACTTCAACAGCGACAC } \\
\text { R: GCCAAATTCGTTGTCATACC }\end{array}$ \\
\hline HMGA2 & siRNA & CAAGAGGCAGACCUAGGAAdTdT (sense) \\
\hline
\end{tabular}

pathological examination. Informed consent was obtained from each patient who provided a specimen, and the research protocol was approved by the Ethics Committee of Huashan Hospital.

Cell culture and reagent. HEK-293T, GBC-SD, EH-GB1 and SGC-996 cells were cultured in Dulbecco's modified Eagle's medium (DMEM) supplemented with $10 \%$ fetal bovine serum (FBS) and $1 \%$ antibiotics.

$R N A$ extraction, reverse transcription and quantitative realtime PCR. Total RNA was extracted from tissues or cells with TRIzol reagent (Invitrogen) according to the manufacturer's protocol. Complementary DNA was synthesized with the PrimeScript RT reagent kit (Takara). Quantitative real-time PCR analyses were performed with SYBR Premix Ex Taq II (Takara). The primers used are listed in Table I. TaqMan microRNA assays (Applied Biosystems) were used to quantify the expression levels of miR-26a.

Oligonucleotide transfection. miR-26a mimics were synthesized by GenePharma. The sequences are 5'-UUCAAGUAAUC CAGGAUAGGCU-3' (sense) and 5'-CCUAUCCUGGAUUA CUUGAAUU-3' (antisense). Small interfering RNAs (siRNAs) targeting HMGA2, E2F7 and PIK3R3 were synthesized by RiboBio. The sequences are listed in Table I. Cells were transfected with oligonucleotides using Lipofectamine 2000 reagent (Invitrogen) at a final concentration of $50 \mathrm{nM}$ and collected for assays at $48 \mathrm{~h}$ post-transfection.

Cell proliferation and colony formation assays. The cell proliferation was measured with the Cell Counting Kit-8 (CCK-8) (Dojindo Laboratories) following the manufacturer's instruc- tion and cell numbers were recorded by the optical density at $450 \mathrm{~nm}\left(\mathrm{OD}_{450}\right)$. For colony formation assay, $1 \times 10^{3}$ cells were plated in each well of a 6 -well plate and incubated at $37^{\circ} \mathrm{C}$ for 2 weeks. Cells were fixed with $4 \%$ paraformaldehyde and stained with $1 \%$ crystal violet (Sigma-Aldrich). Megascopic cell colonies were counted and analyzed.

In vivo tumor formation assay. GBC-SD cells stably expressing miR-26a or vector control were collected and suspended in serum-free DMEM. Each mouse (male BALB/c-nu/nu, 6-weeks old) was injected subcutaneously in the upper back with $2.0 \times 10^{6}$ GBC-SD cells in $200 \mu$ l DMEM. The mice were sacrificed after 6 weeks, and the tumor weight was recorded. The whole experiment was carried out according to the protocols approved by the Shanghai Medical Experimental Animal Care Commission.

Vector constructs. The human pri-miR-26a sequence was amplified from normal human genomic DNA and cloned into the lentivirus expression vector pWPXL (a generous gift from Dr Didier Trono) to generate pWPXL-miR-26a. The 3'-UTR of HMGA2 was amplified and inserted (XhoI + Not I) downstream of the stop codon of Renilla luciferase in psiCHECK2 vector (Promega). The open reading frame (ORF) of HMGA2 was amplified and cloned into another lentiviral vector, pLVX-IRES-Neo (Clontech Laboratories), to generate pLVX-HMGA2. The primers used are listed in Table I.

Lentivirus production and transduction. Lentivirus particles were harvested $48 \mathrm{~h}$ after pWPXL-miR-26a (or pLVX-HMGA2) transfection with the packaging plasmid psPAX2 and VSV-G envelope plasmid pMD2.G (a gift from 

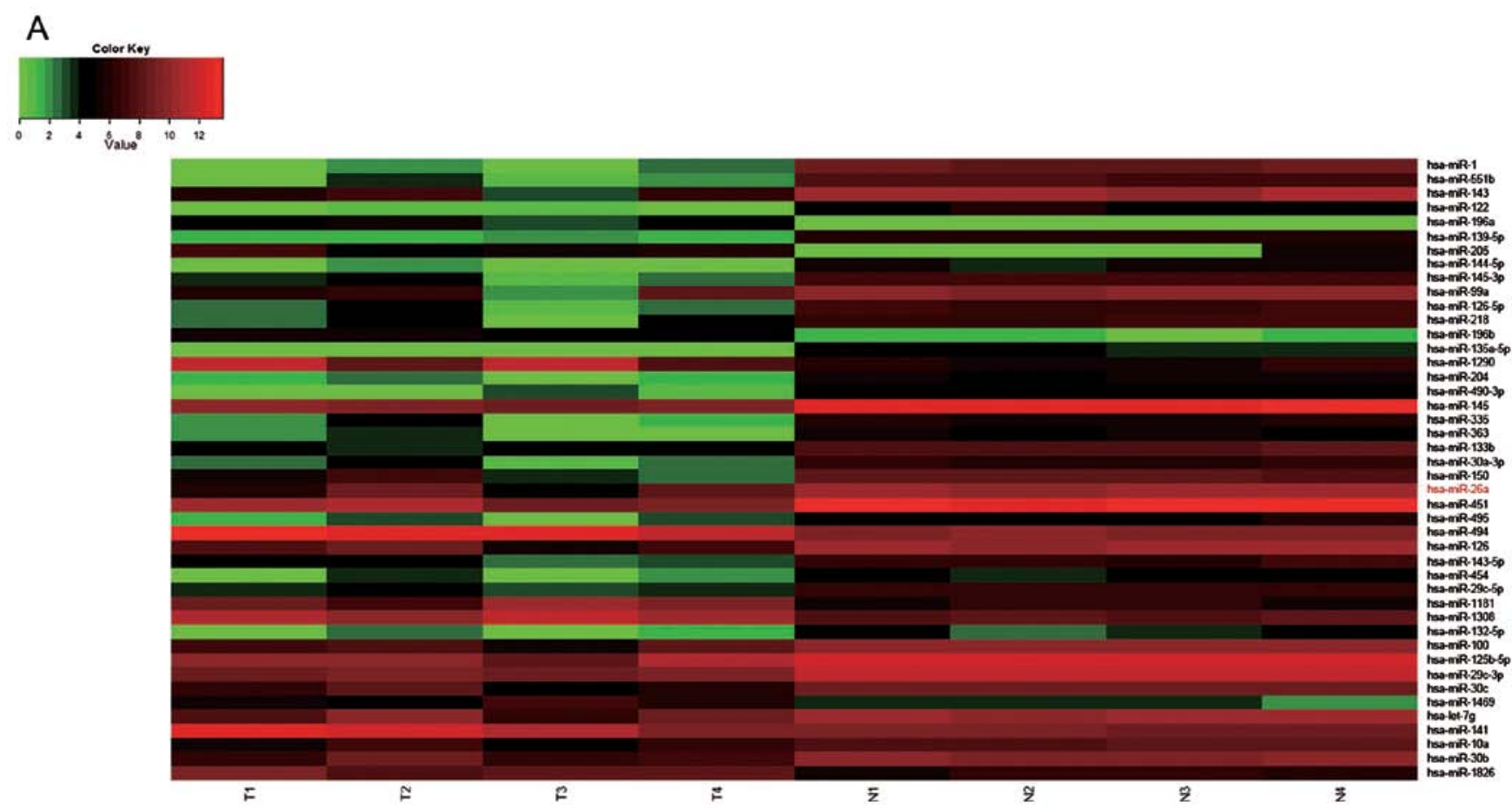

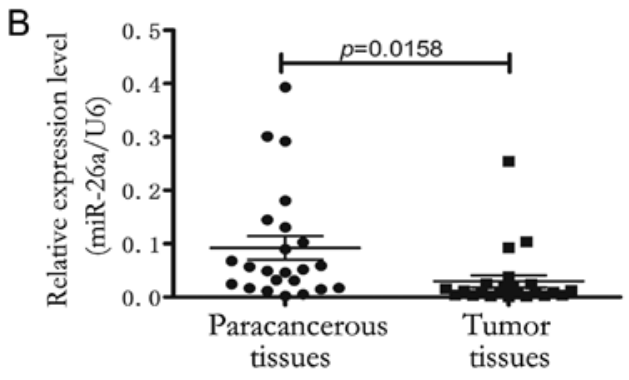

Dr Didier Trono) into HEK293T cells by using Lipofectamine 2000 reagent (Invitrogen). GBC-SD and EH-GB1 cells were infected with recombinant lentivirus plus $6 \mu \mathrm{g} / \mathrm{ml}$ polybrene (Sigma-Aldrich).

Luciferase assay. HEK-293T cells were cultured in 96-well plates and co-transfected with 20 ng psiCHECK-2-HMGA23'-UTR and 5 pmol miR-26a mimic or negative control. After $48 \mathrm{~h}$ of incubation, the firefly and Renilla luciferase activities were measured using the Dual-Luciferase reporter assay system (Promega).

Cell cycle analysis. Cells were collected and fixed in $75 \%$ ethanol at $-20^{\circ} \mathrm{C}$ overnight. The fixed cells were washed three times with phosphate-buffered saline (PBS) and stained with $25 \mu \mathrm{g} / \mathrm{ml}$ propidium iodide (PI) (Kaiji Biotech, Nanjing, China), $10 \mu \mathrm{g} / \mathrm{ml}$ RNase A (Sigma-Aldrich), $0.05 \mathrm{mM}$ ethylene diamine tetraacetic acid (EDTA) and $0.2 \%$ Triton X-100 in PBS for $30 \mathrm{~min}$. DNA content was analyzed with FACSCalibur flow cytometer (BD Biosciences). The results were analyzed using ModFit software (BD Biosciences).

Western blot analysis. Proteins were separated on $10 \%$ SDS-PAGE and transferred to nitrocellulose membrane (BioRad Laboratories). Then the membrane was blocked with
$5 \%$ non-fat milk and incubated with rabbit anti-E2F7 antibody (Abgent) (1:400), rabbit anti-PI3 kinase p85 (PIK3R3) antibody (C-term) (Abgent) (1:400), mouse anti-GAPDH antibody (Sigma-Aldrich) $(1: 3,000)$, mouse anti-Rb (4H1) (Cell Signaling Technology) (1:1,000), rabbit anti-Phospho-Rb (Ser807/811) antibody (Cell Signaling Technology) (1:1,000), rabbit anti-Phospho-Rb (Ser780) antibody (Cell Signaling Technology) (1:1,000), and rabbit anti-HMGA2 antibody (Cell Signaling Technology) $(1: 1,000)$. The proteins were detected with enhanced chemiluminescence reagents (Thermo Fisher Scientific).

Statistical analysis. The results are shown as the means and standard deviation (SD). The data were analyzed with Student's t-test (two-tailed); The clinical significance of miR-26a was analyzed by the way of Fisher's exact test in GraphPad Prism 5. P $<0.05$ was considered to indicate a statistically significant result.

\section{Results}

miR-26a is frequently downregulated in GBC and associated with the histologic grade of the neoplasm. From the results of miRNA expression profiling chip in 4 paired GBC and paracancerous tissues, we found that the miR-26a was 
Table II. The clinical significance of miR-26a.

\begin{tabular}{lcccc}
\hline & miR-26a high & miR-26a low & Total & P-value \\
\hline Age (years) & & & & \\
$>55$ & 4 & 3 & 11 & 0.0995 \\
$\leq 55$ & 8 & 11 & 23 & \\
Total & 12 & & & \\
Gender & & 7 & 16 & \\
Male & 9 & 4 & 7 & 0.6668 \\
Female & 3 & 11 & 23 & \\
Total & 12 & & & \\
Grade & & 2 & 12 & \\
I+II & 8 & 9 & 11 & 0.0361 \\
III+IV & 4 & 11 & 23 & \\
Total & 12 & & & \\
CA199 & & 7 & 12 & \\
High & 5 & 4 & 11 & 0.4136 \\
Low & 7 & & 23 & \\
Total & 12 & & & \\
\hline
\end{tabular}

obviously downregulated (Fig. 1A). And then, we detected mature miR-26a in 23 pairs of GBC and paracancerous tissues and GBC cell lines by real-time PCR. The results confirmed that miR-26a expression was obviously downregulated in GBC tissues compared with the paired paracancerous tissues (Fig. 1B). Based on the 23 paired GBC and paracancerous tissues, the miR-26a expression was associtated with the neoplasm histological grade (Table II).

miR-26a inhibits $G B C$ cell proliferation in vitro and in vivo. The expression of miR-26a is downregulated in GBC and in pancreatic, nasopharyngeal, breast and lung cancer, miR-26a has been shown to act as a tumor suppressor to inhibit tumor cells proliferation by directly targeting EZH2 (9-12), therefore we hypothesized that miR-26a might have influence on GBC proliferation.

To examine the biological function of miR-26a in GBC, we established miR-26a stably expressing cells. Both GBC-SD cells and EH-GB1 cells were infected with the lentivirus containing the miR-26a expression sequence, and the expression of miR-26a was confirmed by real-time PCR (Fig. 2). The cell proliferation of GBC-SD and EH-GB1 infected with lenti-miR-26a was significantly decreased compared with those infected with lenti-GFP based on the CCK- 8 and colony formation assays (Fig. 3A and B). We investigated the effect of miR-26a in cell cycle progression by fluorescence-activated cell sorting analysis. It is shown that the miR-26a blocked G1/S transition in both GBC-SD and EH-GB1 cells (Fig. 7A). Since the expression of miR-26a was extremely low in all GBC cell lines, we did not use the miR-26a silencing method for functional experiments (Fig. 2).

To further determine the effect of miR-26a in GBC cell proliferation in vivo, the GBC-SD cells stably expressing miR-26a or GFP were subcutaneously injected into nude mice.

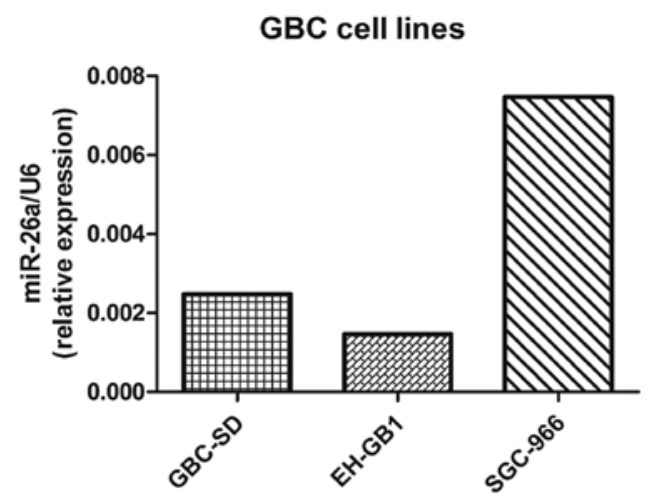

EH-GB1

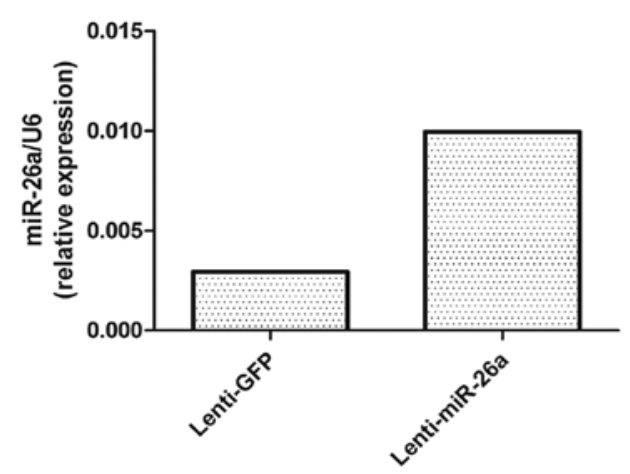

GBC-SD

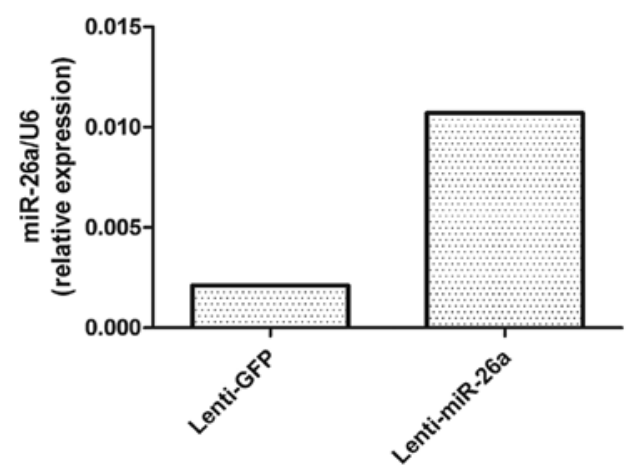

Figure 2. The expression level of miR-26a in GBC cell lines. The miR-26a expression level in the cells used in the present study is presented.

After 6 weeks, the mice were sacrificed to weigh the tumors. The tumor weight of GBC-SD cells stably expressing miR-26a was significantly lower than that of the stably expressing GFP (Fig. 3C).

The above results indicate that miR-26a inhibited the proliferation of GBC cells both in vitro and in vivo.

HMGA2 is a direct downstream target of miR-26a. To identify the direct downstream target responsible for the effect of miR-26a on GBC cell proliferation, mRNA microarray assays were carried out to find the genes that were downregulated by miR-26a in EH-GB1 cells infected with lenti-miR-26a or lentiGFP. Potential targets were predicted by using TargetScan (http://www.targetscan.org), miRanda (http://www.mirandaim.org) and PicTar (http://pictar.mdc-berlin.de). By integrating the results of the four strategies, 13 genes were found to be potential targets of miR-26a (Fig. 4A). In these 13 genes, phosphoinositide-3-kinase, regulatory subunit 3 (gamma) 
A
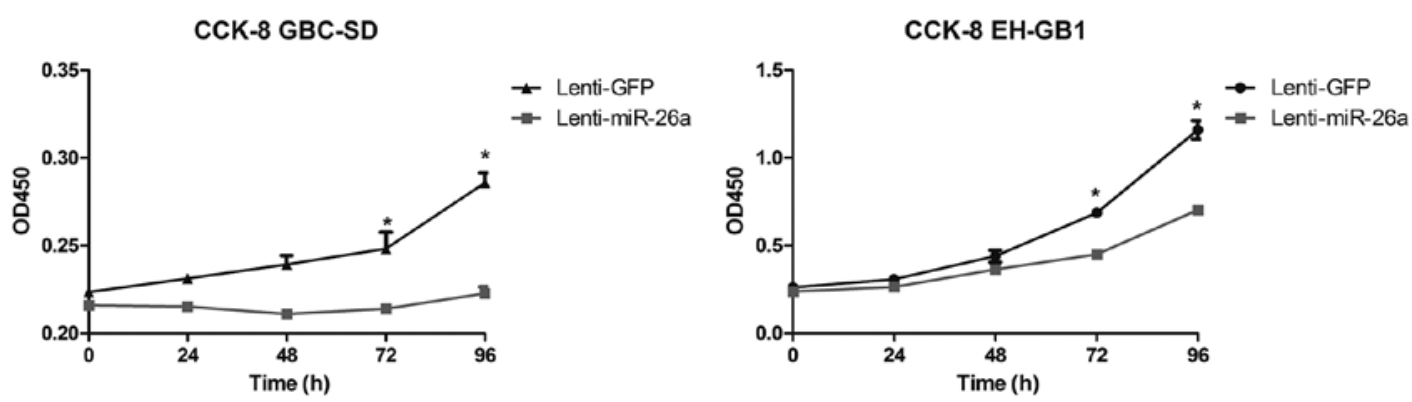

B

Colony formation assays Lenti-GFP Lenti-miR-26a
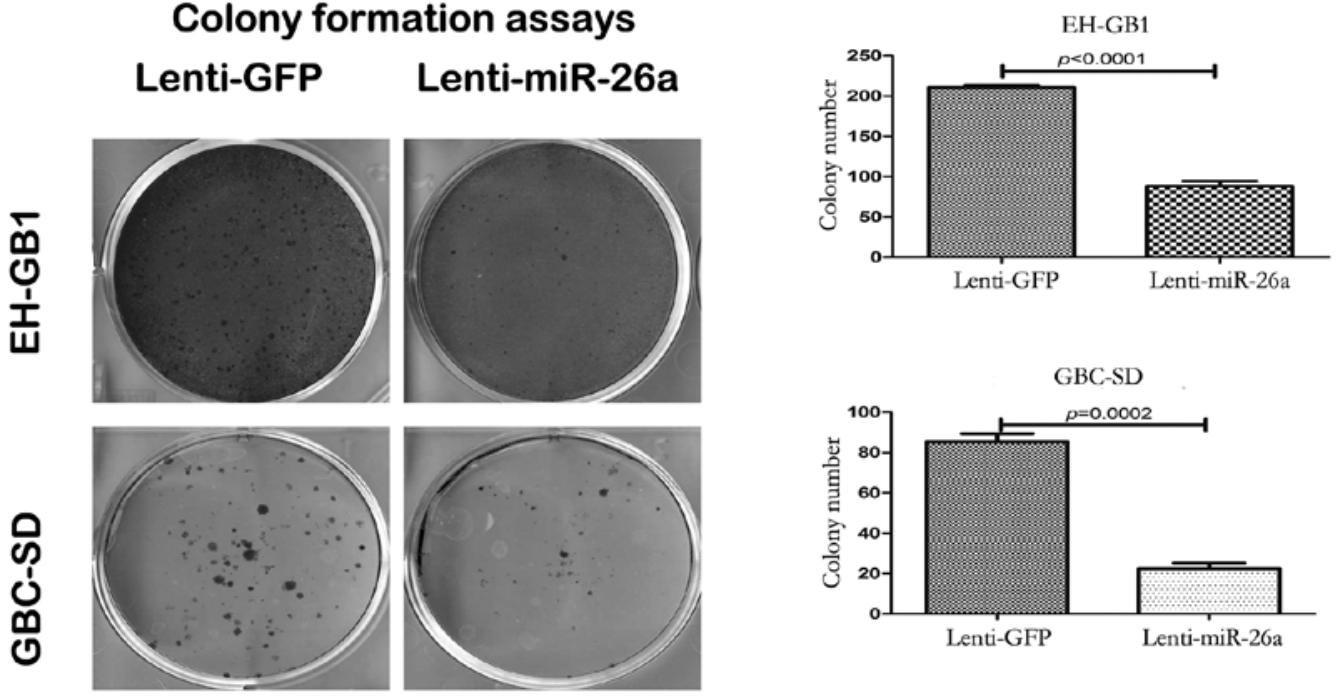

C
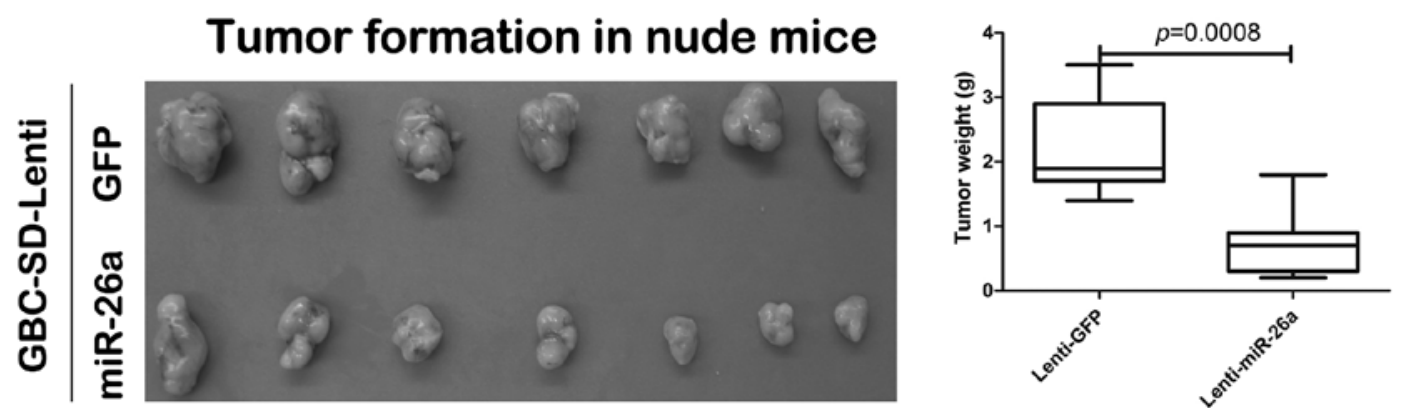

Figure 3. miR-26a inhibits GBC cell proliferation in vitro and in vivo. (A and B) CCK-8 and colony formation assays of GBC-SD and EH-GB1 cells stably expressing miR-26a or GFP control. "P $<0.05$ (Lenti-GFP; Lenti-miR-26a). (C) Tumor formation in nude mice with the GBC-SD cells stably expressing miR-26a or GFP control and the tumor weight.

(PIK3R3), E2F transcription factor 7 (E2F7) and high mobility group AT-hook 2 (HMGA2) have been reported as having effects on the cell cycle inhibiting cell proliferation (16-21). By CCK-8 and colony formation assays, we found that the siRNA against PIK3R3 or E2F7 could not significantly inhibit proliferation of EH-GB1 cell compared to NC, but the proliferation of both EH-GB1 and GBC-SD cells transfected with siRNAs targeting HMGA2 was significantly inhibited compared to that transfected with NC (Fig. 5).

TargetScan indicated that HMGA2 contains 3 miR-26a binding sites on its $3^{\prime}$-UTR, and the sequence of the binding site is highly conserved in different species (Fig. 4C). We constructed vectors containing wild-type or mutant 3'-UTR of
HMGA2 directly inserting downstream of the firely luciferase gene (Fig. 4C), and cotransfected wild-type or mutant vector into HEK-293T cells with miR-26a expression plasmid or vector control. The result showed that miR-26a could significantly decrease the relative luciferase activity of wild-type HMGA23'-UTR, while the luciferase activity in mutant HMGA2-3'-UTR was not reduced as much as in wild-type HMGA2-3'-UTR (Fig. 4B). It is suggested that miR-26a could directly bind to the 3'-UTR of HMGA2. Furthermore, it was found that the HMGA2 mRNA level and miR-26a level was negatively correlated in the 23 pairs of GBC and paracancerous tissues (Fig. 4D). In addition, the HMGA2 protein levels were often higher in GBC tissues than in the paired paracancerous tissues (Fig. 4E). Taken 
A

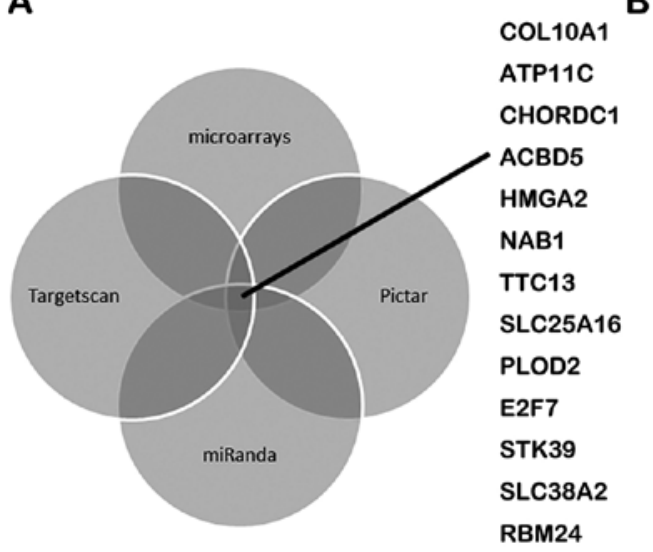

C

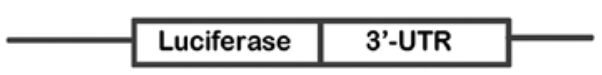

B

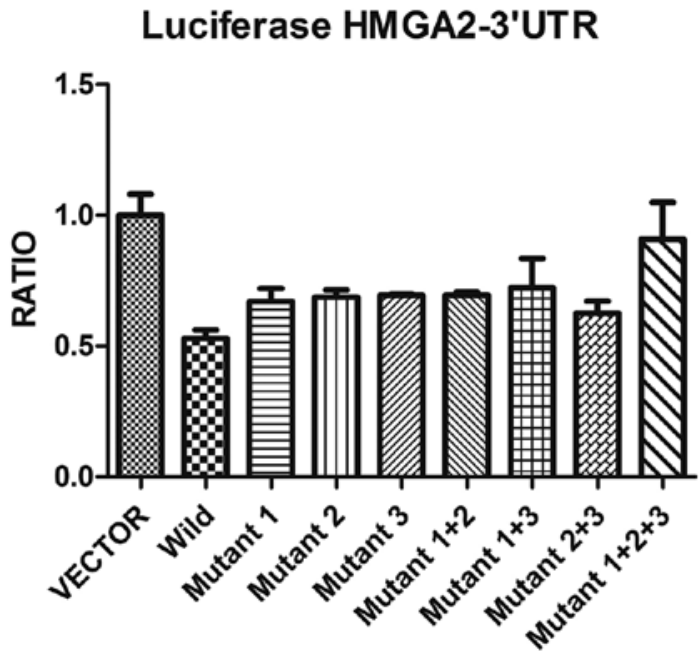

D

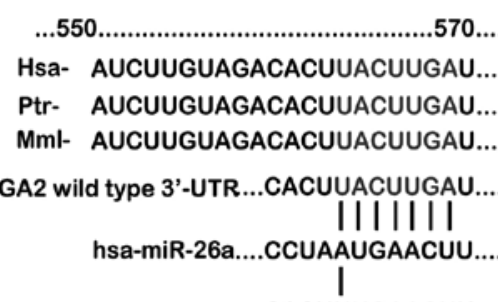

HMGA2 mutant 3'-UTR...CACUUUGAACUU mutant-1

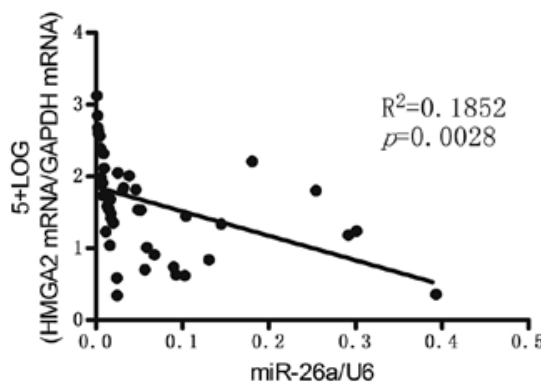

E .AUUAACUUGAAA....................AAAGAAACUUGAAG.. .AUUAACUUGAAA....................AAAGAAACUUGAAG.. ..AUUAACUUGAAA...................CAAAGAAACUUGAAG.. AUUAACUUGAAA ..................CAAAGAAACUUGAAG.. IIIIII AUUAUGAACUAA..................CAAAGAAUGAACUAG. mutant-2 mutant-3

Figure 4. HMGA2 is the direct target of miR-26a. (A) Search for the identification of potential genes combining microarray assays, TargetScan prediction, miRanda prediction and picTar prediction. (B) Relative luciferase activity analyses. The pLUC, pLUC-wild-type-HMGA2-3'-UTR or pLUC-mutant-HMGA23'-UTR was transfected into HEK-293T cells with pWpXL or pWpXL-miR-26a. Renilla luciferase vector was used as an internal control. Representative experiments are shown as the mean \pm SD. (C) The putative binding site for miR-26a within human (Hsa), chimpanzee (Ptr) and rhesus (Mml) HMGA2-3'-UTR, the binding site mutant of HMGA2-3'-UTR was indicated with light gray. (D) Regression and correlation analysis of miR-26a and HMGA2 mRNA in tissues. (E) The HMGA2 protein level is often downregulated in tumor tissues.

together, these results indicate that HMGA2 is a direct downstream target for miR26a in GBC cells.

Reintroduction of HMGA2 abrogates miR-26a-induced proliferation suppression. If HMGA2 indeed is a functional target of miR-26a in GBC cells, reintroduction of HMGA2 without 3'-UTR into miR-26a expressing GBC cells should be able to antagonize the effects of miR-26a. In order to test this hypothesis, we constructed a lentiviral expression vector of HMGA2 ORF (open reading frame) without 3'-UTR and infected miR-26a stably expressing GBC cells with this vector. Western blot assay after HMGA2 ORF lentivirus infection, showed that the protein of HMGA2 expression recovered (Fig. 7B). CCK-8 and colony formation assays demonstrated that reintroduction of HMGA2 antagonized the inhibition of miR-26a to GBC cell proliferation (Fig. 6). Furthermore, enforced expression of HMGA2 counteracted the G1 arrest induced by miR-26a (Fig. 7A). HMGA2 can bind the complex formed by $\mathrm{pRB}$ and HDAC1 to dissociate $\mathrm{pRB}$ from genomic promoter regions and promotes the transcriptional activity of E2F1 and blocks the G1/S transition to inhibit the cell proliferation. In addition (22), we detected the protein level of $\mathrm{Rb}$ and its phosphorylation level in the GBC cells infected with GFP lentivirus, miR-26a lentivirus or both miR-26a lentivirus and HMGA2 lentivirus. The results showed that in both GBC-SD and EH-GB1 cells, Rb protein level was negatively correlated with HMGA2 protein level but its phosphorylation level was positively correlated with HMGA2 (Fig. 7B).

In summary, these results suggest that the HMGA2 is a functional target of miR-26a in GBC cells. 
A

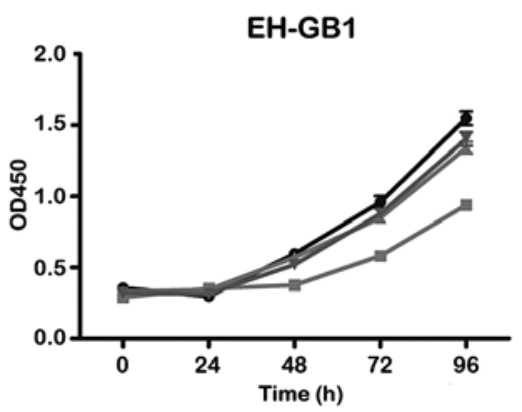

EH-GB1

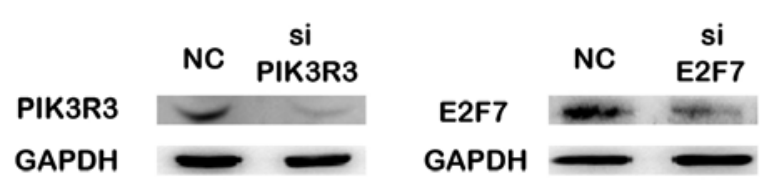

B
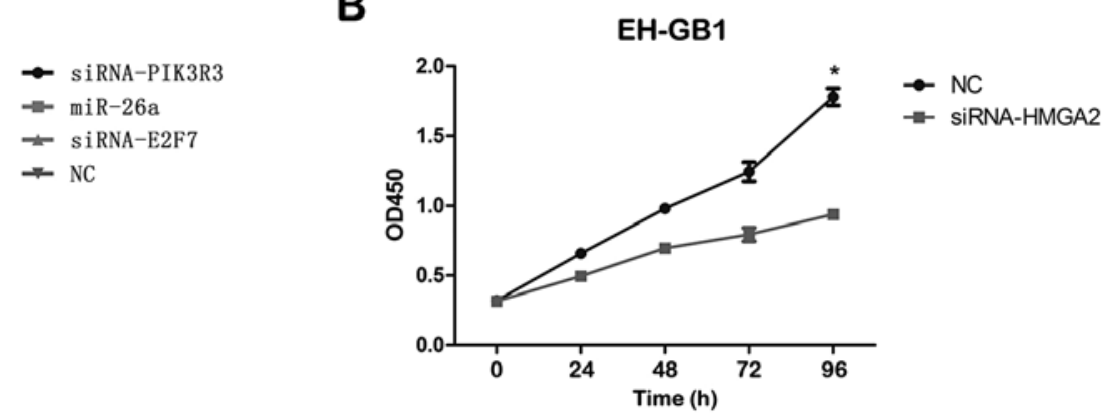

EH-GB1

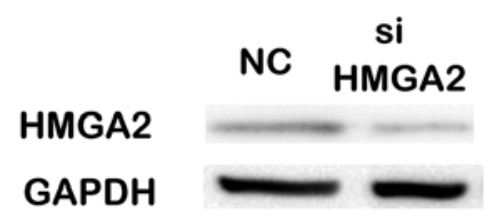

Figure 5. The influence of PIK3R3, E2F7 and HMGA2 silencing on the GBC cell proliferation. (A) CCK-8 assays of EH-GB1 cells transfected with siRNA against PIK3R3 (siRNA-PIK3R3), siRNA against E2F7 (siRNA-E2F7) or negative control (NC). PIK3R3 or E2F7 silencing had no influence to the GBC cell proliferation. (B) CCK-8 assays of EH-GB1 cells transfected with siRNA against HMGA2 (siRNA-HMGA2) or negative control (NC). HMGA2 silencing inhibited the GBC cell proliferation.

A

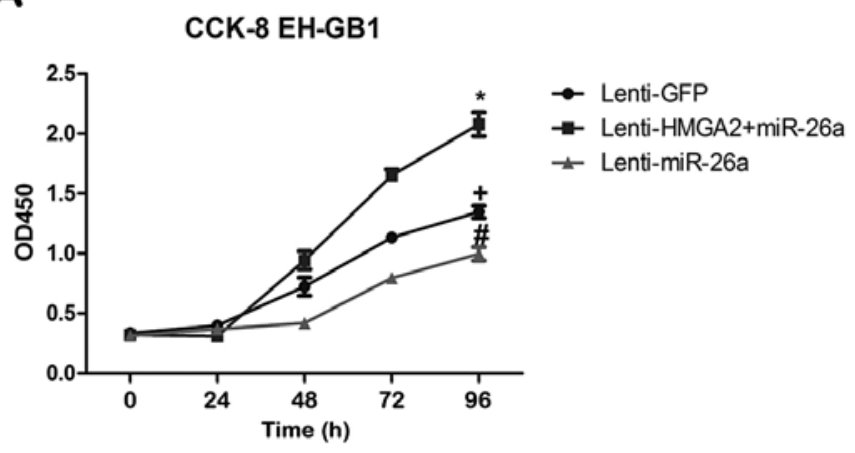

B

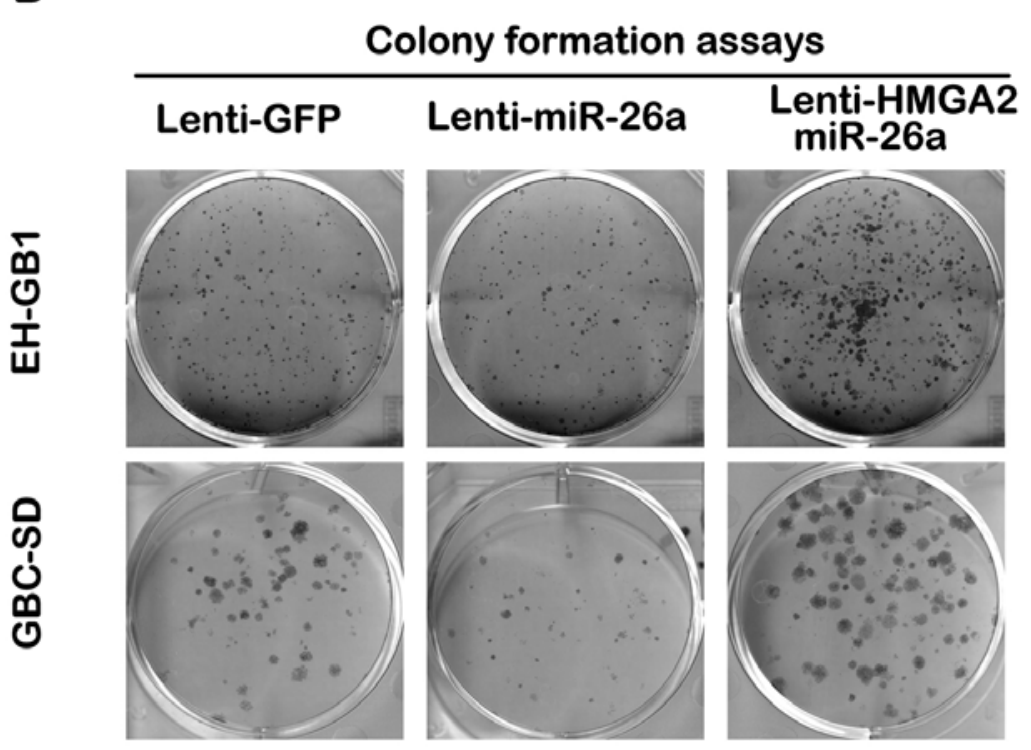

CCK-8 GBC-SD

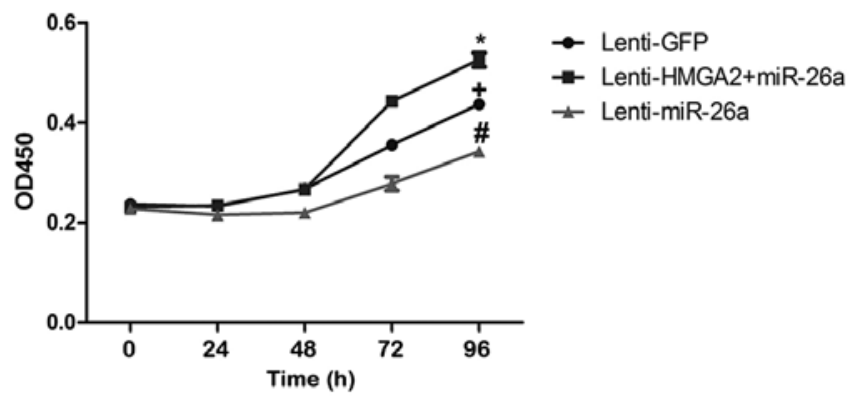

Figure 6. Reintroduction of HMGA2 abrogates miR-26a induced inhibition to poliferation in GBC cells. (A and B) CCK-8 and colony formation assays of GBC-SD and EH-GB1 cells stably expressing miR-26a or lenti-GFP were infected with lenti-HMGA2 or corresponding lenti-GFP. "P<0.05 (Lenti-GFP; Lenti-miR-26a); ${ }^{+}$P<0.05 (Lenti-GFP; Lenti-HMGA2+miR-26a); ${ }^{\prime}($ Lenti-miR-26a; Lenti-HMGA2+miR-26a). 
A

Lenti-GFP Lenti-miR-26a
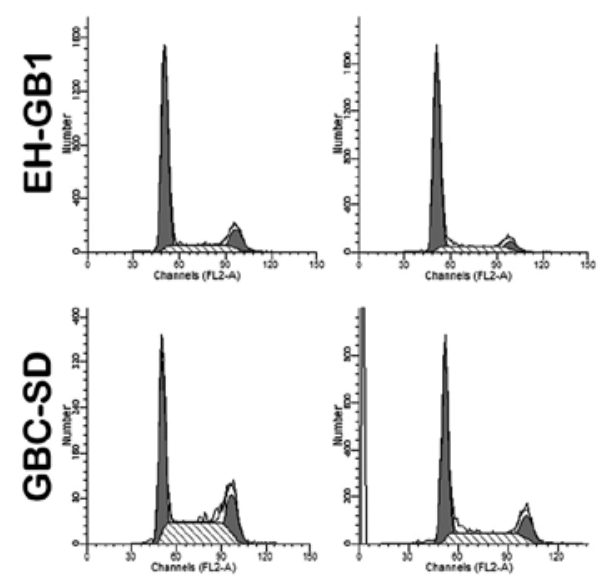

B

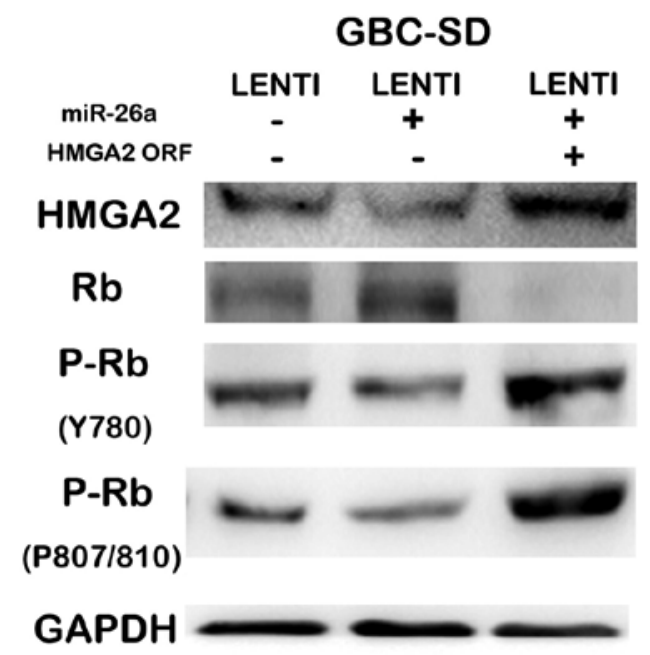

.
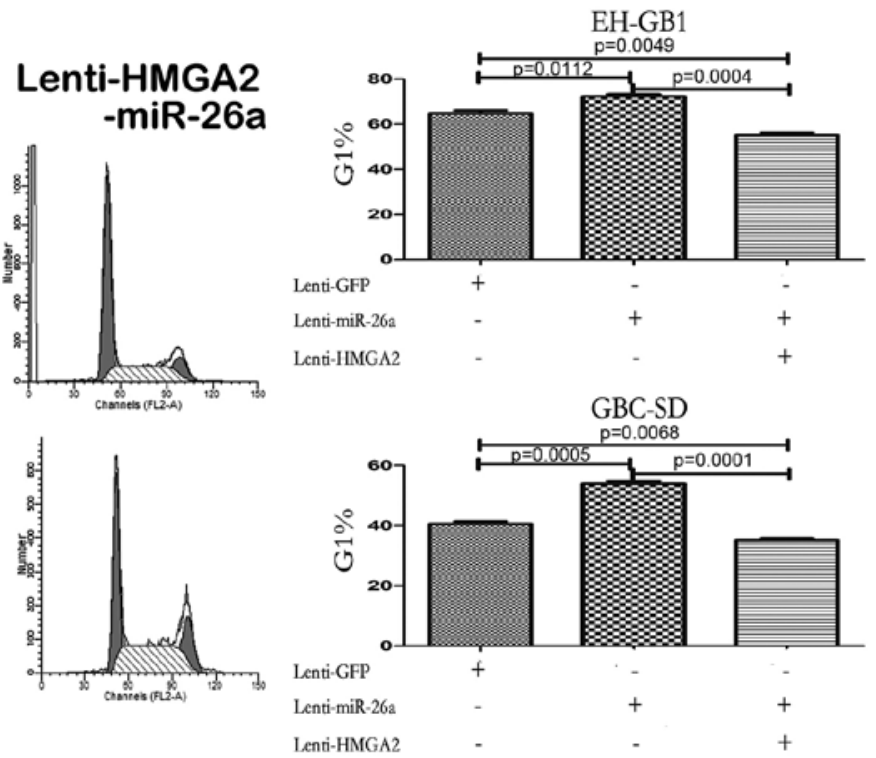
human neoplasm, but it can be not detected in normal somatic cells (21). HMGA2 acts as an activator or a repressor of target gene expression and facilitates DNA structural changes by the formation of the specialized nucleoprotein structures at special promoter regions (24). It is reported that HMGA2 binds the complex formed by $\mathrm{pRB}$ and HDAC1 to dissociate $\mathrm{pRB}$ from genomic promoter regions and promotes the transcriptional activity of E2F1 and blocks the G1/S transition to inhibit the cell proliferation (22). It has been discovered that HMGA2 is upregulated in many benign and malignant tumors, such as colorectal, breast, pancreatic, ovarian, and lung cancer (25-28). In the present study, it was found that the mRNA and protein levels of HMGA2 were often upregulated in GBC tissues. We also found that the HMGA2 siRNA inhibited the proliferation of GBC cells and enforced HMGA2 protein expression promoted the proliferation of GBC cells. In GBC samples, the levels of miR-26a and HMGA2 mRNA were inversely associated, which suggested the upregulation of HMGA2 may be partially due to the downregulation of miR-26a. Furthermore, we found the G1 arrest could be promoted by enforcing miR-26a expression and the arrest by enforcing HMGA2 protein expression. Then, we confirmed the effect by detecting the protein level of $\mathrm{RB}$ and $\mathrm{pRB}$. It is suggested that the HMGA2 is the direct and functional target of miR-26a and involved the miR-26a effect on the proliferation of GBC cells.

Collectively, we found that miR-26a, which is associated with the neoplasm histologic grade, is often downregulated in GBC and it can inhibit the proliferation of GBC cells by directly targeting HMGA2 through G1 arrest, thus miR-26a shows potential as a therapeutic in GBC patients.

\section{Acknowledgements}

We thank Dr Didier Trono (School of Life Sciences, Ecole Polytechnique Fédérale de Lausanne, 1015 Lausanne, Switzerland) for providing the psPAX2,pMD2.G and pWPXL plasmids. The present study was supported by a grant from the Science and Technology Commission of Shanghai Municipality (no. 12nm0501502 and no. 11nm0503900).

\section{References}

1. Bartel DP: MicroRNAs: genomics, biogenesis, mechanism, and function. Cell 116: 281-297, 2004.

2. Bartel DP: MicroRNAs: target recognition and regulatory functions. Cell 136: 215-233, 2009.

3. Croce CM and Calin GA: miRNAs, cancer, and stem cell division. Cell 122: 6-7, 2005.

4. Friedman RC, Farh KK, Burge CB, et al: Most mammalian mRNAs are conserved targets of microRNAs. Genome Res 19: 92-105, 2009.

5. Lewis BP, Burge CB and Bartel DP: Conserved seed pairing, often flanked by adenosines, indicates that thousands of human genes are microRNA targets. Cell 120: 15-20, 2005.

6. Hernando E: microRNAs and cancer: role in tumorigenesis, patient classification and therapy. Clin Transl Oncol 9: 155-160, 2007.

7. Negrini M, Ferracin M, Sabbioni S, et al: MicroRNAs in human cancer: from research to therapy. J Cell Sci 120: 1833-1840, 2007.
8. Chen YL, Huang ZQ, Zhou NX, et al: Clinical analysis of 110 patients with primary gallbladder carcinoma. Zhonghua Zhong Liu Za Zhi 29: 704-706, 2007 (In Chinese).

9. Bao B, Ali S, Banerjee S, et al: Curcumin analogue CDF inhibits pancreatic tumor growth by switching on suppressor microRNAs and attenuating EZH2 expression. Cancer Res 72: 335-345, 2012.

10. Lu J, He ML, Wang L, et al: MiR-26a inhibits cell growth and tumorigenesis of nasopharyngeal carcinoma through repression of EZH2. Cancer Res 71: 225-233, 2011.

11. Zhang B, Liu XX, He JR, et al: Pathologically decreased miR-26a antagonizes apoptosis and facilitates carcinogenesis by targeting MTDH and EZH2 in breast cancer. Carcinogenesis 32: 2-9, 2011.

12. Dang X, Ma A, Yang L, et al: MicroRNA-26a regulates tumorigenic properties of EZH2 in human lung carcinoma cells. Cancer Genet 205: 113-123, 2012.

13. Kota J, Chivukula RR, O'Donnell KA, et al: Therapeutic microRNA delivery suppresses tumorigenesis in a murine liver cancer model. Cell 137: 1005-1017, 2009.

14. Yang X, Liang L, Zhang XF, et al: MicroRNA-26a suppresses tumor growth and metastasis of human hepatocellular carcinoma by targeting interleukin-6-Stat3 pathway. Hepatology 58: 158-170, 2013.

15. Slaby O, Redova M, Poprach A, et al: Identification of MicroRNAs associated with early relapse after nephrectomy in renal cell carcinoma patients. Genes Chromosomes Cancer 51: 707-716, 2012

16. Xia X, Cheng A, Akinmade D, et al: The N-terminal 24 amino acids of the p55 gamma regulatory subunit of phosphoinositide 3-kinase binds $\mathrm{Rb}$ and induces cell cycle arrest. Mol Cell Biol 23: 1717-1725, 2003.

17. Deng Q, Wang Q, Zong WY, et al: E2F8 contributes to human hepatocellular carcinoma via regulating cell proliferation. Cancer Res 70: 782-791, 2010.

18. Sozzani R, Maggio C, Giordo R, et al: The E2FD/DEL2 factor is a component of a regulatory network controlling cell proliferation and development in Arabidopsis. Plant Mol Biol 72: 381-395, 2010.

19. Hazar-Rethinam M, Cameron SR, Dahler AL, et al: Loss of E2F7 expression is an early event in squamous differentiation and causes derepression of the key differentiation activator Sp1. J Invest Dermatol 131: 1077-1084, 2011.

20. Sirma H, Kumar M, Meena JK, et al: The promoter of human telomerase reverse transcriptase is activated during liver regeneration and hepatocyte proliferation. Gastroenterology 141: 326-337, 2011.

21. Li O, Vasudevan D, Davey CA, et al: High-level expression of DNA architectural factor HMGA2 and its association with nucleosomes in human embryonic stem cells. Genesis 44: 523-529, 2006

22. Fusco A and Fedele M: Roles of HMGA proteins in cancer. Nat Rev Cancer 7: 899-910, 2007.

23. Ashar HR, Chouinard RA Jr, Dokur M, et al: In vivo modulation of HMGA2 expression. Biochim Biophys Acta 1799: 55-61, 2010.

24. Eda A, Tamura Y, Yoshida M, et al: Systematic gene regulation involving miRNAs during neuronal differentiation of mouse P19 embryonic carcinoma cell. Biochem Biophys Res Commun 388: 648-653, 2009

25. Ahmed KM, Tsai CY and Lee WH: Derepression of HMGA2 via removal of ZBRK1/BRCA1/CtIP complex enhances mammary tumorigenesis. J Biol Chem 285: 4464-4471, 2010.

26. Cleynen I and Van de Ven WJ: The HMGA proteins: a myriad of functions (Review). Int J Oncol 32: 289-305, 2008.

27. Sgarra R, Zammitti S, Lo Sardo A, et al: HMGA molecular network: from transcriptional regulation to chromatin remodeling. Biochim Biophys Acta 1799: 37-47, 2010.

28. Wang X, Liu X, Li AY, et al: Overexpression of HMGA2 promotes metastasis and impacts survival of colorectal cancers. Clin Cancer Res 17: 2570-2580, 2011. 